\title{
Influential measures on log-normal model for left-truncated and case-k interval censored data with time-dependent covariate
}

\begin{abstract}
In this study, we focus on assessing the performance of the local influential diagnostics on detecting observations that may have an influence on the set of parameters of the extended lognormal distribution which incorporates time-dependent covariates in the presence of lefttruncation and case-k interval censoring. A simulation study was conducted to evaluate the performance of the local influential diagnostics under the proposed perturbation schemes and compared with the global influential diagnostics. The most desirable influential diagnostics are expected to identify influential observations with higher probability when the level of contamination is high.
\end{abstract}

Keyword: Local influential diagnostics; Left-truncation; Time-dependent covariates; Case-k interval censoring 